\title{
Beyond bathymetry - coastal marine mapping
}

\author{
Travis Mason, UK \\ Channel Coastal Observatory \\ Robert KINNEAR, UK \\ Channel Coastal Observatory \\ Andrew COLENUTT, UK \\ New Forest District Council \\ André COCUCCIO, UK \\ The Maritime and Coastguard Agency \\ David PARKER, UK \\ United Kingdom Hydrographic Office \\ Topic F: careful marine planning

\section{INTRODUCTION}

Although IHO Order 1a is now the default standard for the UK's Civil Hydrography Programme surveys, there is a wealth of additional uses of the survey data, which is now being put to good use under the principle of "collect once, use many times". Recent involvement of maritime Local Authorities in England has encouraged extension of the survey into the inter-tidal area, where possible, and to make use of the bathymetry data and backscatter for a range of coastal engineering and management applications.

\section{BACKGROUND}

Coastal surveys have traditionally been difficult since they are usually, by definition, in very shallow water and are heavily tidally- and weather-restricted. In the UK, spring tidal range can vary from 1 to $12 \mathrm{~m}$; the higher ranges can aid the nearshore survey, although where these occur over relatively flat seabed, the speed of the tidal translation can still be a operational problem, whilst near the tidal nodes, the very small tidal range and nearshore bar systems can restrict how close to the shore even a small survey vessel can approach. Furthermore, breaking waves are also a potential hazard close inshore. Taken together, this means that bathymetry surveys of the nearshore require daylight hours, spring tides, good weather, calm seas and a shallow draught vessel; requirements which translate into "expensive" and since the nearshore region is typically of less interest for safety of navigation, the area within $0.5-1 \mathrm{~km}$ of the shoreline is justifiably known as the "White Ribbon", where little data exist.

Since 2008, however, thanks to collaboration with the Maritime Coastguard Agency (MCA)'s Civil Hydrography Programme, the DEFRA-funded, maritime Local Authority-led Southeast and Southwest Regional Coastal Monitoring Programmes have been able to upgrade their bathymetry survey programme from single-beam to swath bathymetry, with a resultant explosion in the usefulness of the data. The first collaborative project was between the MCA, New Forest District Council and Dorset Wildlife Trust and, together with a grant from Viridor Credits, resulted in the "DORIS" survey, covering some $800 \mathrm{~km}^{2}$ from the MLWS contour to $6 \mathrm{~nm}$ offshore, from Portland Bill to Swanage. Subsequently, further similar collaboration via the lead Local Authorities of the Southeast, Southwest and East Riding of Yorkshire Regional Coastal Monitoring Programmes means that $20 \%$ of the coastal strip of England has been surveyed to IHO Order $1 \mathrm{a}$, with plans to cover a further $5 \%$ within the next 2 years.

Although not the responsibility of maritime Local Authorities, the recent introduction of the UK's Marine Bill, and subsequent requirement for the creation of Marine Conservation Zones and Maritime Protected Areas has resulted in a burst of interest in coastal surveys to provide data for mapping of marine habitats, based on the interpretation of the swath bathymetry and backscatter. 


\section{METHODS}

The swath bathymetry data commissioned by the Regional Coastal Monitoring Programmes is used for mapping habitats based on the JNCC version of the European-wide EUNIS classification system. Three broad parameters in varying combinations are used for EUNIS Levels I to III, in order of increasing complexity (Level I merely distinguishes between marine, freshwater and terrestrial habitats). Firstly, the nature of the seabed itself, which can be either rock or sediment or rock with a covering of thin sediment. The second banding is based on photic levels i.e. the depth to which light can penetrate, which depends essentially on the water clarity. The shallowest band is the inter-tidal region and hence the elevation of the Mean Low Water Springs contour can be used as the boundary between the shallowest (Littoral) and middle (Infralittoral) depth bands; in the English Channel, $5 \mathrm{~m} \mathrm{CD}$ is a typical depth boundary to the deepest category used for shelf seas (Circalittoral).

The third discrimination parameter is energy-based; this is not overly sophisticated being either High (e.g. Land's End, Cornwall), Medium (central English Channel coast) or Low (eastern Kent) wave energy. However, there may be areas within an overall Low wave energy category which are reclassified as Medium energy due to the presence of strong tidal currents, for example Hurst Narrows in the Solent. Combinations of these parameters produce 8 marine habitat classifications at EUNIS Level II and 26 categories at Level III. Finally, a revised substrate classification is then produced by reducing the Level III categories to rock, coarse or mixed sediment, sand or mud.

Amongst some of the habitat mapping community, there is an impression at large that all the mapping is, or can be, done directly from the backscatter, but accurate habitat mapping relies on a combination of bathymetry, backscatter and ground-truthing, with the relative importance of each facet varying with substrate. For example, on exposed, rock platforms, the habitat can be mapped almost entirely from the bathymetry alone (including derived information such as slope and rugosity) whilst, in contrast, rock substrate covered with a layer of thin sediment, or discrimination between sand and mud require careful interpretation of the backscatter and, ideally, ground-truth information such as grab sampling or video.

During the mapping procedures to produce EUNIS classification, GIS shape files are produced for anthropogenic feature such as pipelines, obstructions and wrecks, scour marks, trenches and cables. Slope, rugosity and, in some cases, geological information such as fault lines can also be mapped and stored as shape files for subsequent further investigation. From a coastal management point of view, the advantage of a Level III classification is the detailed mapping of the seabed substrate including classes of mud, sand, coarse or mixed sediment.

\section{RESULTS AND DISCUSSION - Seabed mapping for coastal management}

The seabed mapping results given here are from coastal bathymetric surveys conducted by maritime Local Authorities in conjunction with the MCA and UKHO. All data and mapping results are made freely available at www.channelcoast.org.

\section{Marine habitat mapping}

Until the relatively recent use of swath bathymetry and simultaneous backscatter, much of the habitat mapping in the UK was derived from modelling, validated in some cases by widely-spaced strips of multibeam data, but with grab sampling and biological sampling. As a result, the confidence in the results was high for the ground truthing assessment but low as regards to coverage and positioning. In contrast, habitat mapping from the IHO Order 1a surveys gives very high confidence estimates due to the $100 \%$ coverage of the seabed and accurate positioning, which usually result into a high overall confidence score despite the limited ground truthing. Biological sampling is still required to achieve the more detailed Levels IV and V but a Level III habitat map is still highly useful given the sparcity of measured data for defining draft Marine Conservation Zone areas. For example, seabed mapping of a 
recent swath bathymetry survey off Kent allowed the extent of the chalk reef (an EC Habitats Directive Annex I habitat) to be accurately mapped for the first time, along with the other marine habitats (Figure 1).

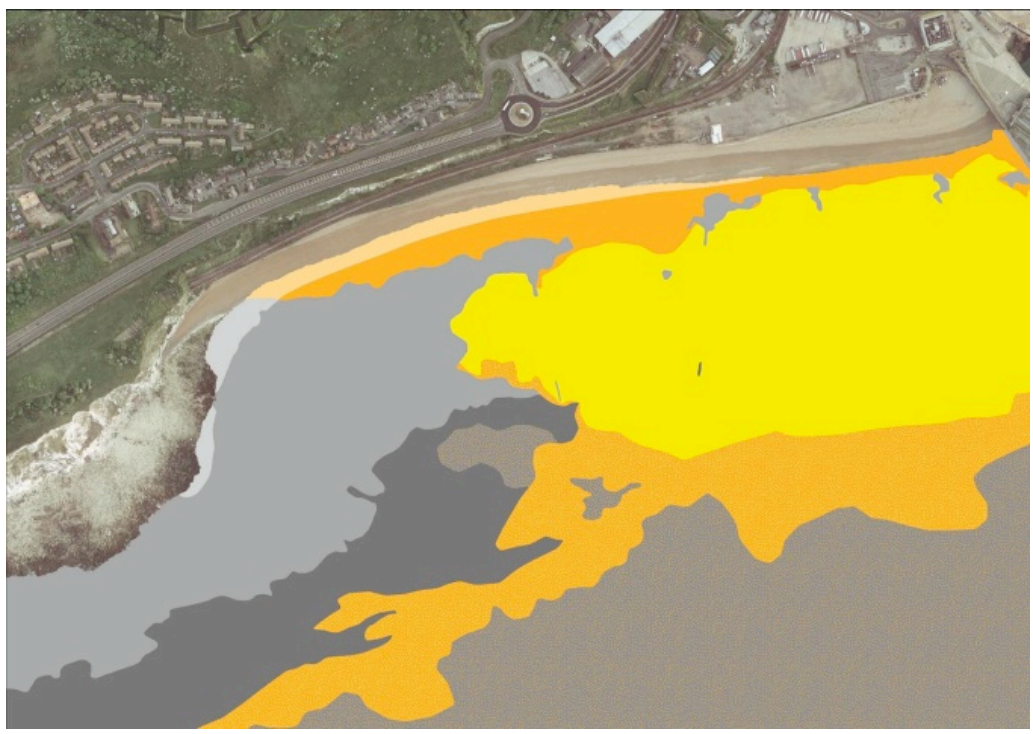

Figure 1: EUNIS Level III habitat map, just south of Dover, Kent. The bathymetry overlaps the inter-tidal beach captured by ortho-rectified photography so that a seamless coastal habitat map can be achieved

LEGEND: EUNIS Level 3

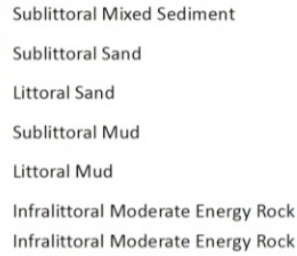

Infralittoral Moderate Energy Rock Infralittoral Moderate Energy Rock and thin Sediment

\section{Coastal engineering}

In most cases, design of coastal structures or beach management operations are based on singlebeam profiles $50-100 \mathrm{~m}$ apart at best. The additional information from the seabed mapping of swath bathymetry means that the seabed substrate, texture, variability, micro-scale slope and potential obstructions can now be taken fully into account. In Gurnard Bay, Isle of Wight, for example, the presence of long-term pipelines still visible in the bathymetry confirms the suspected lack of "spare" sediment in the system as well as the stability of the seabed (Figure 2). Operationally, the detailed nearshore bathymetry and resultant obstructions/ made-made features mapping have proved invaluable for planning operations to replenish beaches from dredgers.

\section{Figure 2:}

Bathymetric survey of the northwest Isle of Wight, Hampshire, 2011

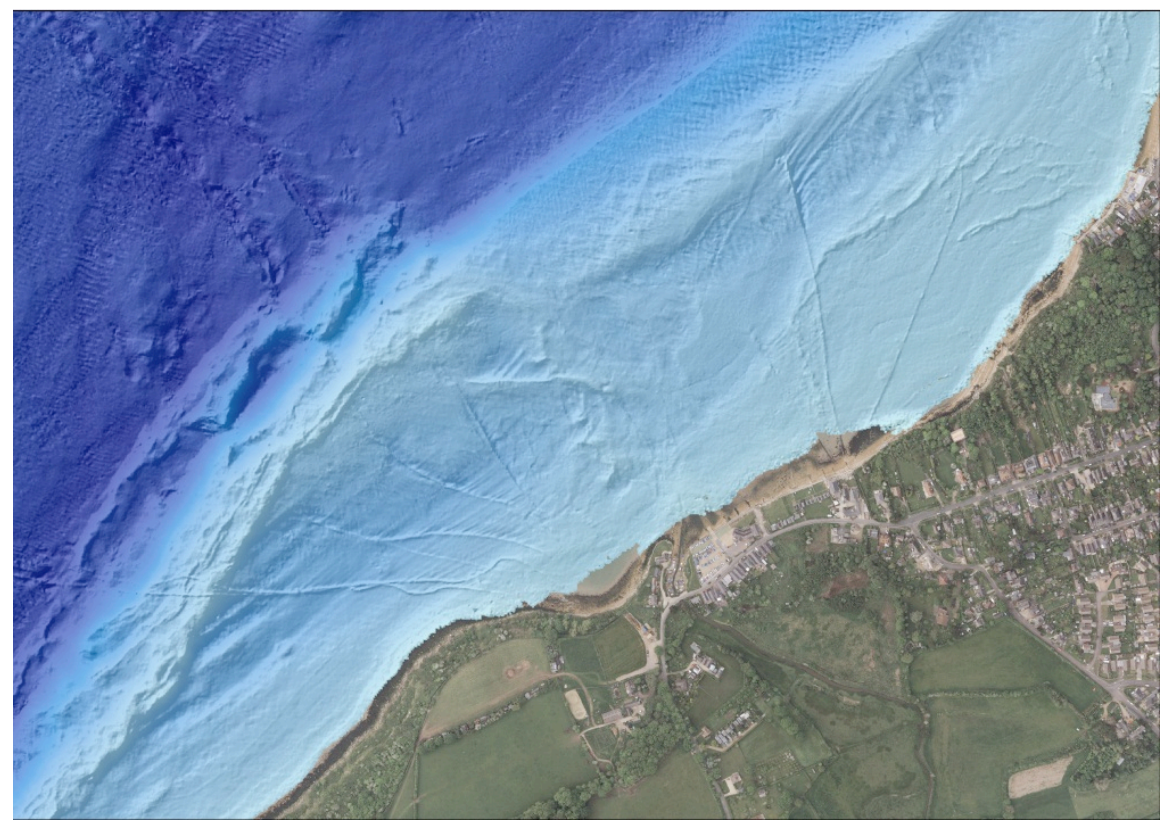




\section{Implications for sediment transport}

Predictions of sediment transport rates are notoriously inaccurate and despite decades of world-wide fieldwork and modelling, a daily sediment transport rate accurate to within 2 orders of magnitude is realistically the best that can be achieved; even predicting net transport direction over the timescales of months or years is fraught with difficulty, let alone for the decade timescales required for governmental planning. Part of the reason for this is that nearly all of the nearshore coastal monitoring takes place in the inter-tidal region. The Regional Coastal Monitoring Programmes of England undertake twice-yearly beach surveys using either RTK GPS topographic surveys or laser scanning and/or airborne lidar, which help to provide a good picture of the annual variability of the sediment. However, using land-based survey techniques means that surveys can extend seawards only to MLWS at best.

Typically, storms draw down beach material to the just sub-tidal area (say MLWS to $-2 m$ CD), but whether it is eventually returned to the beach (the classic summer/winter beach profile pattern) or becomes subject to tidal or wave-induced longshore currents resulting in transport out of the sediment system is rarely known or understood. This is a particular problem on steep shingle beaches, such as Slapton Sands, Devon, or Seaford, East Sussex, where the $~ 1: 8$ beach slope and typically plunging waves prevent land-based surveyors from venturing into the water, and prevent boat surveys from approaching too close inshore. Yet without an assessment of the sub-tidal beach volume, few conclusions can be made regarding the sediment transport patterns implied by topographic difference models. Mapping of the substrate close inshore can help with this problem.

Swath bathymetry in the coastal zone has revealed hitherto unknown detail on sedimentary features such as sandwaves and other bedforms, giving an important source of new information for coastal management (Figure 3). Not only does the very presence of such features indicate a potential subtidal sediment supply, but new semi-automatic tools for calculating crest orientation, wavelength, height and asymmetry direction and ratios developed at the University of Southampton (Cazenave et al., 2012) can provide useful proxies for net direction of sediment transport and thus potentially the relative net effect of tidal and wave regimes. Research is onging to assess whether bedforms are active or relict which, again, will have significant implications for future marine planning.

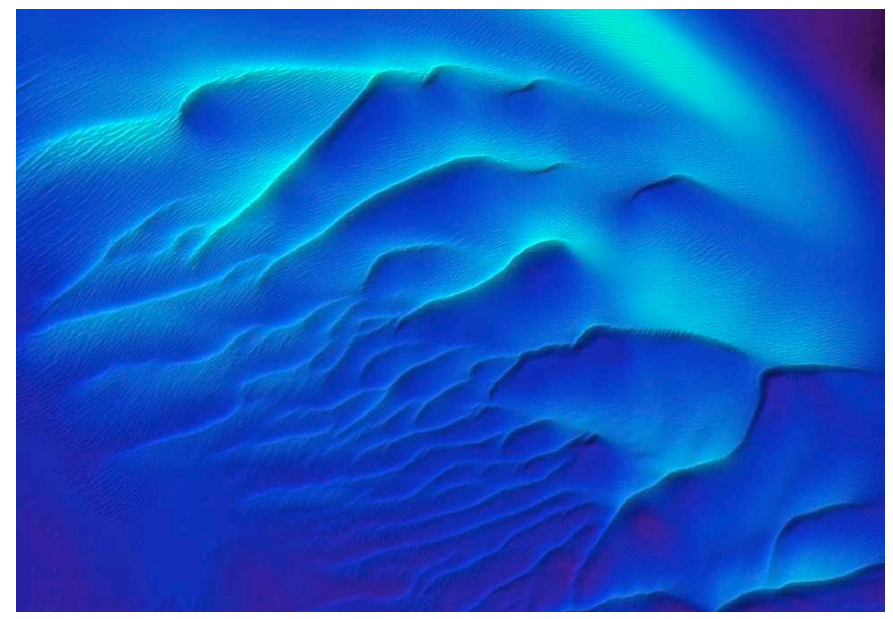

Figure 3: Mobile sand and gravel waves and parasitic bedforms (DORIS survey, 2010)

Since marine sediment is an important source of material for beach recharge, the swath bathymetry provides the opportunity to identify potential new offshore banks in areas of the coastline where beaches are intensively managed, particularly if they can be shown to be non-mobile. Advantages include reduction in mobilisation and transport costs and the likelihood that sediment from within the same sediment circulation regime will be closer to the natural beach material both in terms of composition and sediment size, which is an important aspect in the performance of the recharged beach. 


\section{Design wave conditions}

Most coastal structures or beach management plans involve design wave conditions based on wave heights, periods and direction measured (or more likely, hindcast from wind fields) in 30-50m water depth and numerically transformed to the nearshore location. Wave transformation models rely heavily on nearshore bathymetry, since most of the transformation occurs in water depths shallower than $20 \mathrm{~m}$ and, being a non-linear process, becomes progressively more influential inside $10 \mathrm{~m}$. The more sophisticated spectral wave models e.g. SWAN can resolve wave transformation over quite complex seabeds, taking into account refraction, diffraction and wave shoaling but the results are still dependant on a realistic representation of the seabed over which the transformation is modelled.

For example modelling of the wave climate at Preston Beach, Weymouth, is a particular challenge due to the peninsula of Portland Bill, and similarly the presence of ebb tide deltas off Hayling Island have a marked influence on the wave conditions at the shoreline; the DORIS survey and planned surveys in the eastern Solent are expected to improve the modelling of wave extremes at the coastline, for subsequent improvement of beach management practices.

Implications for future monitoring

Previous coastal monitoring of bathymetry, with single beam profiles at $100 \mathrm{~m}$ intervals, gave no indication of the nature of the seabed and surveys had been repeated several years apart, but were still unsuitable for difference modelling due to the sparse data. The results of the seabed mapping identifies areas of rock, for which there will be no need to re-survey in the foreseeable future, leading to considerable cost savings for coastal monitoring.

\section{CONCLUSIONS}

The more development of multibeam sensors within the last few years, the release by the MCA of a technical specification for IHO Order 1a swath bathymetry surveys, incorporation of backscatter processing and analysis into bathymetric survey software and a willingness by several public bodies in the UK to share resources and subsequent survey data have been major drivers in the uptake of swath bathymetry data for non-traditional uses. Surveys suitable for safety of navigation and charting have valuable secondary uses for coastal engineering and management as well as for habitat mapping and marine spatial planning. In turn, it encourages public bodies in the UK, when conducting bathymetry surveys for non-navigational purposes, to consider specifying Order 1a standard, where possible, so that the surveys are truly multi-purpose.

\section{REFERENCE}

Cazenave, P. W., Dix, J. K., Lambkin, D. O. and McNeill, L. C. (2012). A method for semi-automated objective quantification of linear bedforms from multi-scale digital elevation models. Earth Surface Processes and Landforms. doi: 10.1002/esp.3269

\section{ACKNOWLEDGEMENTS}

The seabed mapping was conducted under the auspices of the UK's MAREMAP project. The technical guidance of Dr Tim Le Bas (National Oceanography Centre) and Dr Justin Dix (University of Southampton) is acknowledged, with thanks, as is the assistance of Kent Wildlife Trust.

\section{BIOGRAPHIES}

Travis MASON is an oceanographer, specialising in coastal hydrodynamics and sediment transport. She is responsible for the SE and SW Regional Coastal Monitoring Programmes' extensive coastal 
wave and tide network in southern England, and also takes the lead for the introduction of Order 1a bathymetry to the new National Network of Coastal Monitoring Programmes of England.

Robert KINNEAR is a coastal process scientist undertaking marine habitat mapping of the Regional Coastal Monitoring Programmes' swath bathymetry data.

Andrew COLENUTT is a Project Manager specialising in flood and coastal erosion risk management. He is also responsible for technical oversight of the interpretation and production of marine habitat and substrate mapping of the swath bathymetry collected through the Regional Coastal Monitoring Programmes.

André COCUCCIO is a marine geoscientist specialising in seabed survey and mapping to underpin safety of navigation in UK waters. He is the head of hydrography at the UK Maritime and Coastguard Agency, the principal hydrographer for the UK Civil Hydrography Programme and director of the European seabed mapping project "INIS Hydro".

David PARKER has worked in hydrography on every continent, including Antarctica, but within UK waters he is responsible for managing the UKHO's involvement in the Civil Hydrography Programme, in particular planning survey areas and defining technical requirements.

\section{CONTACT DETAILS}

\section{Travis MASON}

Channel Coastal Observatory

National Oceanography Centre

European Way

Southampton

UK

Tel.: +442380598467

Fax: +44 2380516320

Email: Travis.Mason@noc.soton.ac.uk

Web site: www.channelcoast.org 